\title{
REGULAR AND STOCHASTIC DYNAMICS OF RELATIVISTIC PARTICLES IN ALFVÉN WAVES
}

\author{
S. M. CARIOLI ${ }^{1}$ and V. N. FEDORENKO ${ }^{2}$ \\ ${ }^{1}$ Max-Planck-Institut für Kernphysik, Heidelberg, FRG. \\ ${ }^{2}$ A. F. Joffe Physico-Technical Institute, Leningrad, USSR.
}

\begin{abstract}
We study the exact phase space dynamics of relativistic test particles propagating in static one-dimensional Alfvén waves, modelling cosmic ray propagation in the interplanetary medium and in the interstellar medium. The result shows that the conventional approach should not be considered adequate to explain important features of particle propagation in Alfvén waves.
\end{abstract}

Our present understanding of cosmic ray propagation and acceleration is mainly based on a kinetic approach, the well known quasilinear theory of particle transport in magnetic turbulence ${ }^{1}$. We wanted to be in a position to verify the domain of validity of quasilinear theory and to solve problems which lie outside this domain. We set up a Hamiltonian formulation of the basic problem of test particles propagating in Alfvén waves and used the methods of dynamical system theor ${ }^{4}$ in order to find the solution. Our basic physical assumptions are the following: we neglect collisions, radiation losses and any transfer of energy and momentum to the waves. We consider only Alfvén waves. The mean magnetic field is constant in space and time and the wave field depends only on the coordinate $z$ along the direction of the mean magnetic field. These assumptions single out the simplest nontrivial physical system investigated so far by means of the methods of quasilinear theory ${ }^{3}$. This system can be considered as a model of particle propagation in the static interstellar medium ${ }^{5}$.

The physical picture of particle transport in Alfvén waves underlying quasilinear theory is the process of resonant wave-particle interaction. This process has been investigated by many authors in the context of dynamical system theory ${ }^{4}$. A resonant wave-particle interaction is a genuine nonlinear process leading to a very strong interaction of a given particle with only one harmonic of the field. In the context of quasilinear theory the resonance condition is given by cyclotron resonance. The pitch angle cosine, which is exactly preserved in the absence of waves, is considered as a zero order adiabatic invariant. The first order correction is affected by a secular divergence due to the cyclotron resonance. This fact is interpreted as the destruction of the adiabatic invariant, leading to a random walk of the particle in phase space and therefore to a Fokker-Planck equation ${ }^{1}$.

The first point is that only the primary resonance is considered. That is, a particle is defined to be in resonance with a wave when the Larmor frequency is equal to the 
frequency of the wave in the particle proper frame. Now, resonances occur when at least two eigenfrequencies of the unperturbed system are commensurable and not just equal. Thus, secondary and higher order resonances are completely neglected in quasilinear theory. Indeed, the resonant wave-particle interaction is the result of the collective effect of all the resonances between the motion of a particle and the given wave $e^{4}$.

The second point is that a resonance is realized by a periodic orbit of the particle. A periodic orbit may be either stable or unstable. If the resonant orbit is stable the adiabatic invariant is not destroyed in the vicinity of the resonance but instead achieves a different topology ${ }^{4}$. This implies that the orbits near the stable equilibrium realizing the resonance are closed whilst the orbits that are far from the resonance are open curves like the zero order orbits. This is the only reason why one obtains a small divisor for the first order correction. The appropriate treatment of this problem ${ }^{2}$ delivers the correct adiabatic invariant that is not destroyed in the vicinity of the primary resonance (Fig. 1). This arises because when the wave and the particle are in resonant interaction the primary cyclotron resonance is realized by a stable periodic orbit. In other words, the only resonance accounted for in the quasilinear calculations traps the particles instead of scattering them.
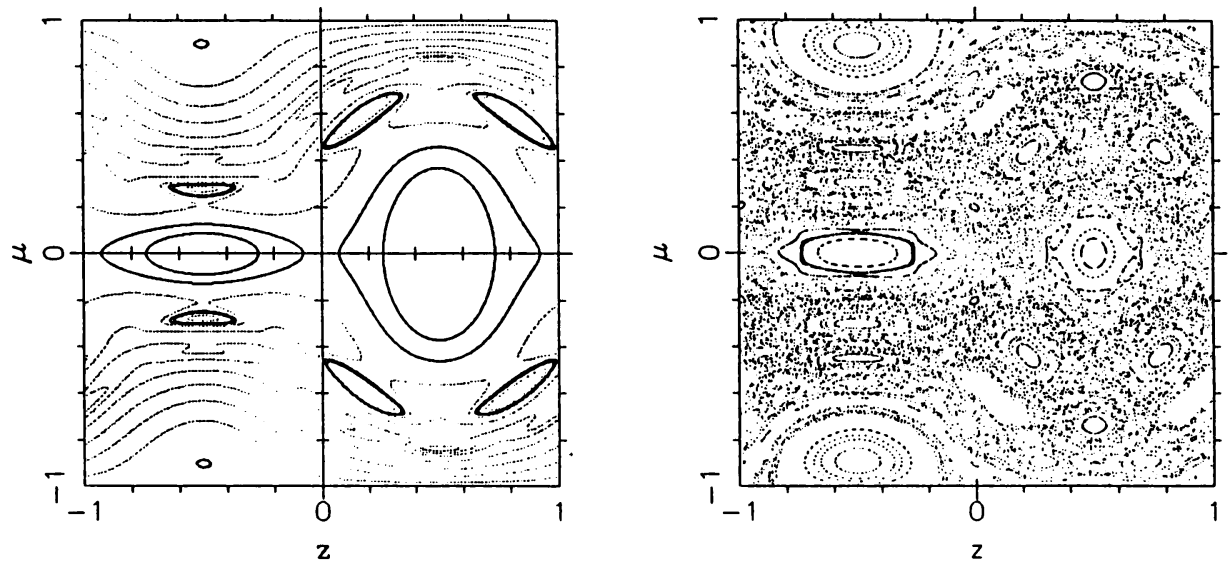

Figure 1. We calculated the approximate invariant for a proton of $9 \mathrm{GeV} / \mathrm{c}$ moving in a linearly polarized monochromatic wave of relative amplitude $\delta B / B_{z}=0.3$ and wavelength $\lambda=10 c \frac{2 \pi}{\Omega}$, where $\boldsymbol{\Omega}$ is the Larmor frequency.

Figure 2. The exact Hamiltonian flow was obtained numerically in the same case. The pitch angle cosine is defined as $\mu=p_{z} / p$. The coordinate $z$ is normalized to $\lambda / 2$.

\section{References}

${ }^{1}$ Blandford, R. D., and Eichler, D.: 1987, Phys. Reports 154, 1.

${ }^{2}$ Carioli, S. M., and Fedorenko, V. N.: 1989, in preparation.

${ }^{3}$ Fedorenko, V. N.: 1987, Astrofizika 26, 285.

${ }^{4}$ Lichtenberg, A. J., and Lieberman, M. A.: 1983, Regular and Stochastic Motion, Springer.

${ }^{5}$ Völk, H. J.: 1975, Rev. Geophys. Space Phys. 13, 547. 